\title{
The lived experience of moving forward after spinal cord injury: a study using Parse research method Hsiao-Yu Chen
}

\author{
Address: Central Taiwan University of Science and Technology, Department of Eldercare, Taiwan \\ from $5^{\text {th }}$ International Conference on Conservative Management of Spinal Deformities \\ Athens, Greece. 3-5 April 2008 \\ Published: 15 January 2009 \\ Scoliosis 2009, 4(Suppl I):P4 doi:I0.II86/I748-7|6I-4-SI-P4
}

This abstract is available from: http://www.scoliosisjournal.com/content/4/SI/P4

(C) 2009 Chen; licensee BioMed Central Ltd.

\begin{abstract}
Aim
The purpose of this study is to discover the structure of the ("lived") real life experience of moving forward after sustaining a spinal cord injury using the Parse research method. Another goal is to better understand the impact of the Taiwanese culture on this experience. The overall goal is to improve the quality of life for patients with spinal cord injury.
\end{abstract}

\section{Methods}

This study was conducted in a Rehabilitation Hospital and Spinal Injury Association in the middle of Taiwan. Participants were 15 clients with spinal cord injury. The Parse research method was used to answer the research question: what is the structure of the lived experience of moving forward? The Parse research method illuminates the structure of lived experiences of health, such as moving forward, through the processes of dialogical engagement, extraction-synthesis and heuristic interpretation.

\section{Results}

The central finding of this study is the following structure: the lived experience of moving forward is a triumphing over handicaps, an ability to transition thoughts with an open mind, an emergence of self-worth based on one's faith.

\section{Discussion}

The findings are discussed in relation to human becoming, related literature and future research. The concept of client-centered care is discussed.

\section{References}

I. Chen HY, Boore JRP: Living with a spinal cord injury: a grounded theory approach. Journal of Clinical Nursing 2008, 17:116-124.
2. Gatehouse M: Moving forward: the guide to living with spinal cord injury. London: Spinal Injuries Association (SIA); 1995.

3. Parse RR: The Human Becoming school of thought: a perspective for nurses and other health professionals. London: SAGE Publications; 1998. 